\title{
GENERATION OF PROPER CLASSES OF SHORT EXACT SEQUENCES
}

\author{
ALI PANCAR \\ Department of Mathemancs \\ Karadeniz Tech. University \\ 61080 - Trabzon / TURKEY \\ (Received September 18, 1995 and in revised form April 10, 1996)
}

\begin{abstract}
The generation of proper classes of short exact sequences of modules by subclasses is considered. The class generated by two proper classes is studied by means of some operations between these classes These operations are investigated in details for classes of short quasi-splitting, torsionsplitting and pure exact sequences of abelian groups.
\end{abstract}

KEY WORDS AND PHRASES: Proper class of short exact sequences, generated proper class, coprojectively (coinjectively) generated class, sum of proper classes, quasi-splitting (pure, torsion splitting) short exact sequences, cotorsion groups, Ulm's subgroup.

1991 AMS SUBJECT CLASSIFICATION CODES: 18G25, $20 \mathrm{~K} 40$.

\section{INTRODUCTION}

By module we mean a unitary left $R$-module, $R$ being an associative ring with identity and by group we mean a $\mathbf{Z}$-module, i.e. an abelian group.

Let $a$ be a class of short exact sequences of modules. If a short exact sequence

$$
\mathrm{E}: 0 \longrightarrow \mathrm{A} \stackrel{\alpha}{\longrightarrow} \mathrm{B} \stackrel{\beta}{\longrightarrow} \mathrm{C} \longrightarrow 0
$$

belongs to $Q, \alpha$ is said to be an $Q$-monomorphism and $\beta$ an $Q$-epimorphism. A short exact sequence $E$ is determined by each of the monomorphism $\alpha$ and epimorphism $\beta$ uniquely up to isomorphism. In the case of necessity, without loss of generality, we shall regard any monomorphism to be an inclusion map stands for the end of the proof. We use the terminologies and notations of Fuchs [1] and Mac Lane [2].

DEFINITION. The class $\mathbb{a}$ is said to be proper, if it satisfies the following conditions (see Buchsbaum [3], Mac Lane [2], Sklyarenko [4]):

(P-1) Along with any short exact sequence $\mathfrak{R}$ contains every one isomorphic to it.

$(\mathrm{P}-2) \mathbb{Q}$ contains all splitting short exact sequences.

(P-3) The composite of two $\mathbb{Q}$-monomorphisms is an $\mathbb{Q}$-monomorphism if this composite is defined.

(P-3') The composite of two $\mathbb{Q}$-epimorphisms is an $\mathbb{Q}$-epimorphism if it is defined

(P-4) If $\alpha, \beta$ are monomorphisms and $\beta \circ \alpha$ is an $Q$-monomorphism, then $\alpha$ is an $Q$-monomorphism

$\left(\mathrm{P}-4^{\prime}\right)$ If $\gamma, \delta$ are epimorphisms and $\delta \circ \gamma$ is an $Q$-epimorphism, then $\delta$ is an $Q$-epimorphism.

Short exact sequences of the form (1.1) from $\mathbb{a}$ give the subgroup $\operatorname{Ext}_{\mathbb{R}}(\mathrm{C}, \mathrm{A})$ of the group of extensions $\operatorname{Ext}_{R}^{1}(C, A)$. Throughout this paper we shall write $\operatorname{Ext}(C, A)$ instead of $\operatorname{Ext}_{R}^{1}(C, A)$

DEFINITION. Let $\varepsilon$ be a class of short exact sequences. The least proper class containing $\varepsilon$ is said to be generated by $\varepsilon$ and denoted by $<\varepsilon$.

Since the intersection of any family of proper classes is proper, we have evidently

$$
<\varepsilon=\cap\{\mathbb{Q}: \varepsilon \subseteq \mathbb{R} ; \mathbb{Q} \text { is a proper class }\} .
$$

The notion of a generated proper class is a generalization of projectively (injectively), coprojectively (coinjectively) generated, and other, proper classes (see Stenström[5], Alizade [6]). In 
this paper some properties of the class $<\varepsilon>$ are given in terms of $\varepsilon$ and the structure of this class is investigated by means of some operations between subclasses.

2. GENERATED PROPER CLASSES.

DEFINITION. A module $A$ is called $\mathbb{R}$-coprojective ( $\mathbb{R}$-coinjective) if every short exact sequence ending (beginning) at $A$ belongs to $\mathbb{R}$.

The least proper class for which every module from the class of modules $A$ is coprojective (coinjective) is denoted by $\bar{k}(A)(\underline{k}(A))$. Such classes are sard to be coprojectively (coinjectively) generated (see Alizade [7][6]).

PROPOSITION 2.1. Let $\mathcal{P}$ be a class of modules. For each $P \in \mathcal{P}$ take a short exact sequence

$$
\mathrm{E}^{\mathrm{P}}: 0 \longrightarrow \mathrm{K}_{\mathrm{P}} \longrightarrow \mathrm{F}_{\mathrm{P}} \longrightarrow \mathrm{P} \longrightarrow 0
$$

with some projective $F_{\mathrm{P}}$. Denote $\left\{\mathrm{E}^{\mathrm{P}}: \mathrm{P} \in \mathcal{P}\right\}$ by $\mathcal{E}_{1}^{\boldsymbol{f}}$. Then $\bar{k}(\mathcal{P})=\left\langle\mathcal{E}_{1}^{\boldsymbol{p}}\right\rangle$.

PROOF. Denote $\left\langle\varepsilon_{1}^{f}>\right.$ by $\mathscr{Q}$. Since each module $P \in \mathcal{P}$ is $\bar{k}(\mathcal{P})$-coprojective, every $E^{P}$ from $\varepsilon_{1}^{P}$ belongs to $\bar{k}(\mathbb{P})$. $\mathbb{Q}$ is the least proper class which contains all $\mathrm{E}^{\mathrm{P}}$, therefore $\mathscr{Q} \subseteq \bar{k}(\mathcal{P})$.

On the other hand, since $E^{P} \in \mathbb{Q}$, for any module $A$ we can write the following exact sequences:

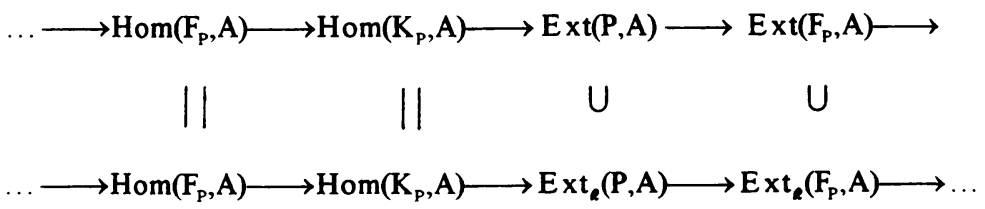

$\mathrm{Ext}_{\mathrm{R}}\left(\mathrm{F}_{\mathrm{p}}, \mathrm{A}\right)=\mathrm{Ext}\left(\mathrm{F}_{\mathrm{p}}, \mathrm{A}\right)=0, \mathrm{~F}_{\mathrm{p}}$ being a projective module. By 5-Lemma $\operatorname{Ext}_{\mathbb{R}}(\mathrm{P}, \mathrm{A})=\operatorname{Ext}(\mathrm{P}, \mathrm{A})$ Then $P$ is an $Q$-coprojective module. Since $\bar{k}(P)$ is the least proper class for which every $P \in \mathcal{P}$ is coprojective, we have $\bar{k}(\mathcal{(}) \subseteq \mathbb{Q}$.

The dual statement can be proved for coinjectively generated classes.

DEFINITION. Let $\mathbb{R}$ be a class of short exact sequences. A module A said to be $\mathbb{R}$-projective ( $Q$-injective), if for every $\mathbb{Q}$-epimorphism ( $\mathbb{Q}$-monomorphism) $\sigma: \mathrm{B} \rightarrow \mathrm{C}$

$$
\sigma_{*}: \operatorname{Hom}(\mathrm{A}, \mathrm{B}) \longrightarrow \operatorname{Hom}(\mathrm{A}, \mathrm{C})\left(\sigma^{*}: \operatorname{Hom}(\mathrm{C}, \mathrm{A}) \longrightarrow \operatorname{Hom}(\mathrm{B}, \mathrm{A})\right)
$$

is an epımorphism. The class of all $\mathbb{Q}$-projective (respectively, $\mathfrak{Q}$-injective) modules is denoted by $\pi(\mathfrak{Q})$ (resp. $\mathrm{t}(\mathbb{Q})$ ) (see [4], [5]).

A proper class $Q$ is said to be projective, if for every module $A$ there is an $Q$-epimorphism from an $Q$-projective module $\mathrm{P}$ onto $\mathrm{A}$. An injective class is defined dually.

PROPOSITION 2.2. Let $\mathscr{R}$ be a projective class and $\varepsilon$ be the class of all short exact sequences

$$
0 \longrightarrow \mathrm{A} \longrightarrow \mathrm{P} \longrightarrow \mathrm{C} \longrightarrow 0
$$

from $\mathbb{R}, \mathrm{P}$ being an $\mathbb{R}$-projective module. Then $\mathbb{Q}=<B$.

PROOF. The inclusion $\angle \mathbb{\subseteq} \subseteq \mathbb{Q}$ is obvious. On the other hand, let (1.1) be an arbitrary short exact sequence from $Q$. Since $\mathscr{Q}$ is a projective class, there exists an $Q$-epimorphism $\gamma: P \rightarrow B$ with an $\mathbb{Q}$-projective $P$. By $\left(\mathrm{P}^{-} 3^{\prime}\right) \beta \circ \gamma$ is an $\mathbb{Q}$-epimorphism At the same time $\beta \circ \gamma$ is an $<\&>$-epimorphism since $P$ is an $Q$-projective module. Therefore, $\beta$ is an $<\varepsilon>$-epimorphism by $\left(P-4^{\prime}\right)$. Thus $E \in<\varepsilon>$.

The proposition dual to proposition 2.2 can be proved for an injective class $Q$

PROPOSITION 2.3. $\pi(\varepsilon)=\pi(<\varepsilon)$.

PROOF. $\varepsilon \subseteq<\varepsilon>$ by the definition of $\langle\varepsilon>$, therefore $\pi(\varepsilon) \supseteq \pi(<\varepsilon>)$. On the other hand, the proper class $\pi^{-1}(\{K\})$ includes $\varepsilon$ for every $K \in \pi(\varepsilon)$. Since $\langle\varepsilon\rangle$ is the least proper class including $\varepsilon$, 
$<B \subseteq \pi^{-1}(\{K\})$. Therefore, $K$ is projective for every short exact sequence from $<B>$ Then $K \in \pi(<\beta)$ Thus $\pi(\varepsilon) \subseteq \pi(<\varepsilon>)$

PROPOSITION 2.4. $t(\varepsilon)=t(<\varepsilon)$.

The proof is dual to that of Proposition 2.3

\section{SOME OPERATIONS BETWEEN PROPER CLASSES.}

DEFINITION. The proper class $Q+\mathcal{L}=\angle \mathbb{Q} \triangleright>$ is called the sum of the proper classes $\mathbb{Q}$ and $\mathcal{L}$

Let us denote the class of short exact sequences whose monomorphisms are $\alpha \circ \beta, \alpha$ being an $Q$-monomorphism and $\beta$ being an $\mathcal{L}$-monomorphism, by $\mathbb{Q} \circ \mathcal{L}$.

Define the class $Q$ * $\mathcal{L}$ of short exact sequences by the formula

$$
\mathrm{Ext}_{\mathrm{R}+\mathrm{L}}(\mathrm{C}, \mathrm{A})=\mathrm{Ext}_{\mathrm{R}}(\mathrm{C}, \mathrm{A})+\mathrm{Ext}_{\boldsymbol{L}}(\mathrm{C}, \mathrm{A}) \text {. }
$$

We have the following obvious facts:

1) $a+\ell=\ell+Q$ is a proper class;

2) $\mathbb{Q} * \mathcal{L}=\ell * \mathbb{Q}$;

3) $\mathfrak{Q} \circ \mathcal{L} \subseteq \ell+\mathbb{Q}$ and $\mathfrak{Q} * \ell \subseteq \mathbb{Q}+\mathcal{L}$.

In Theorem 3.1 we present the situation where $\mathscr{Q} * \mathcal{L} \subseteq \mathbb{R} \circ \mathcal{L}$.

In general $\mathbb{R} \circ \ell$ and $\mathbb{Q} * \ell$ need not be a proper class (see Corollary 4.1) But if $\mathbb{R}_{\circ} \ell$ (or $\mathbb{R} * \ell$ ) is proper then, clearly, $\mathbb{Q}+\mathcal{L}=\mathbb{R} \mathcal{L}(\mathbb{Q}+\mathcal{L}=\mathbb{Q} * \mathcal{L})$ and we can study $\mathbb{Q}+\mathcal{L}$ by means of $\mathbb{R}$ and $\mathcal{L}$ (as in [6]).

The following proposition can be derived from Theorem 2.1 of Kepka [8], but we give another proof of this fact which relies on Thoeorem 1 of Alizade [7].

PROPOSITION 3.1. If the class of modules $p$ is closed under submodules and extensions

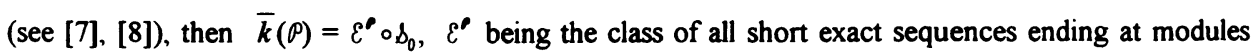
from $\rho$ and $s_{0}$ being the class of all splitting short exact sequences.

PROOF. Take an arbitrary short exact sequence (1.1) from $\bar{k}(P)$. Since $P$ is closed under extensions, by Theorem 1 of Alizade [7] there exist $P \in P, E_{1} \in \operatorname{Ext}(\mathbf{P}, \mathbf{A})$ and a homomorphism $\gamma: \mathbf{C} \rightarrow \mathbf{P}$ such that $E=\gamma^{*}\left(E_{1}\right)$. Since $P$ is closed under submodules, $\operatorname{Im} \gamma \in \mathcal{P}$. Therefore $\gamma$ can be taken as an epımorphism. From the cohomology sequence for $0 \longrightarrow \mathrm{K} \stackrel{\delta}{\longrightarrow} \mathrm{C} \stackrel{\gamma}{\longrightarrow} \mathrm{P} \longrightarrow 0, \mathrm{~K}=\mathrm{Ker} \gamma$.

$$
\ldots \longrightarrow \operatorname{Ext}(\mathrm{P}, \mathrm{A}) \stackrel{r^{*}}{\longrightarrow} \operatorname{Ext}(\mathrm{C}, \mathrm{A}) \stackrel{\delta^{*}}{\longrightarrow} \operatorname{Ext}(\mathrm{K}, \mathrm{A}) \longrightarrow \ldots
$$

we have $\operatorname{E} \in \operatorname{Im} \gamma^{*}=\operatorname{Ker} \delta^{*}$. Then in the following commutative diagram with exact rows and columns

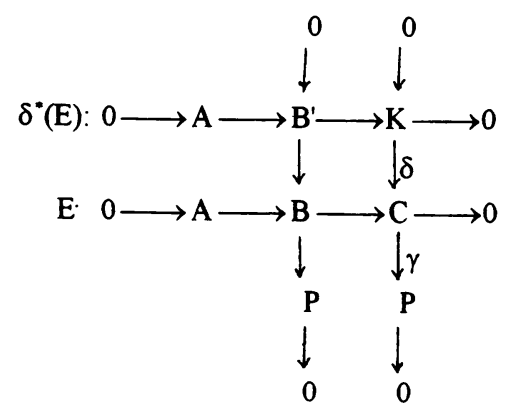

$\delta^{*}(\mathrm{E}) \in \wp_{0}$ On the other hand, since $0 \longrightarrow \mathrm{B}^{\prime} \longrightarrow \mathrm{B} \longrightarrow \mathrm{P} \longrightarrow 0 \in \mathcal{E}^{\boldsymbol{p}}$ we have $\mathrm{E} \in \mathcal{C}^{\boldsymbol{c}} \circ \$_{0}$

Conversely, since $\varepsilon^{f} \subseteq<\mathcal{E}^{f}>$ and $s_{0} \subseteq<\mathcal{E}^{f}>$, we have $\mathcal{E}^{f} \circ \delta_{0} \subseteq<\mathcal{E}^{f}>\subseteq \bar{k}(\mathcal{P})$.

LEMMA 3.1. Let (1.1) be a short exact sequence, $\gamma: D \rightarrow B$ be an arbitrary homomorphism and $F=\gamma^{-1}(A)$. Then the homomorphism $\delta: D / F \rightarrow C$, induced by $\gamma$, is a monomorphism.

PROOF. If $\delta(d+F)=0$ then $\gamma(d) \in A$. Therefore $d \in \gamma^{-1}(A)=F$ and $d+F=F$. 
LEMMA 3.2. If $p$ is a class of modules, closed under extensions and submodules (see [7], [8]), then for $\alpha=\bar{k}(\mathcal{P})$ and any proper class $\mathcal{L}, \mathrm{Ext}_{\mathrm{R}_{\alpha \ell}}$ is a subfunctor of Ext : R-mod $\times \mathrm{R}$-mod $\rightarrow \mathrm{Ab}$, $\mathrm{R}$-mod being the category of left $\mathrm{R}$-modules and $\mathrm{Ab}$ being the category of abelian groups.

PROOF. Let $\mathrm{E}: 0 \longrightarrow \mathrm{A} \stackrel{s}{\longrightarrow} \mathrm{D} \longrightarrow \mathrm{F} \longrightarrow 0 \in \mathrm{Ext}_{\text {eos }}(\mathrm{F}, \mathrm{A})$ and $\gamma: \mathrm{A} \rightarrow \mathrm{M}$ be an arbitrary homomorphism Since $E \in \mathbb{R} \circ \mathcal{L}, \delta=\alpha^{\prime} \circ \beta^{\prime}, \alpha^{\prime}$ being an $\alpha$-monomorphism and $\beta^{\prime}$ being an $\mathcal{L}$-monomorphism. By Prop.3.1 $\alpha^{\prime}$ can be written as $\alpha^{\prime}=\alpha \circ \theta$, where $\theta$ is a splitting monomorphism and $P=D / I m \alpha \in \mathcal{P}$ Therefore $\theta \circ \beta^{\prime}=\beta$ is an $\ell$-monomorphism. Thus $\delta=\alpha \circ \beta$ and we have the following commutative diagram with exact rows and columns:

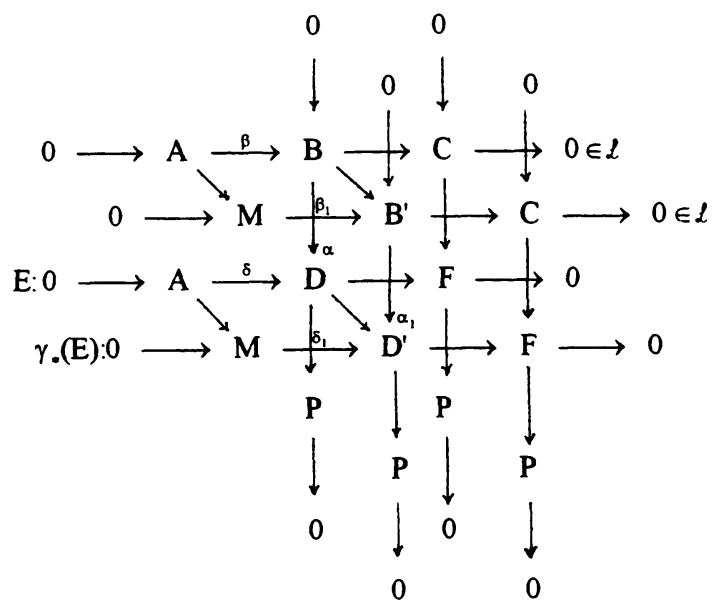

The short exact sequence $0 \longrightarrow \mathrm{M} \stackrel{\beta_{1}}{\longrightarrow} \mathrm{B}^{\prime} \longrightarrow \mathrm{C} \longrightarrow 0$ belongs to $\mathcal{L}$ as an image of the short exact sequence $0 \longrightarrow A \stackrel{B}{\longrightarrow} B \longrightarrow C \longrightarrow 0$ Then $\beta_{1}$ is an $\ell$-monomorphism.

The short exact sequence $0 \longrightarrow \mathrm{B}^{\prime} \stackrel{\alpha_{1}}{\longrightarrow} \mathrm{D}^{\prime} \longrightarrow \mathrm{P} \longrightarrow 0$ belongs to $\mathbb{A}$ as an image of $0 \longrightarrow \mathrm{B} \longrightarrow \mathrm{D} \longrightarrow \mathrm{P} \longrightarrow 0$. Then $\alpha_{1}$ is an $\mathbb{Q}$-monomorphism. Therefore $\delta_{1}=\alpha_{1} \circ \beta_{1}$ is an $\mathbb{Q}_{0} \ell-$-monomorphism and $\gamma_{*}(\mathrm{E}): 0 \longrightarrow \mathrm{M} \stackrel{\delta_{1}}{\longrightarrow} \mathrm{D}^{\prime} \longrightarrow \mathrm{F} \longrightarrow 0 \in \mathbb{R} \circ \mathcal{L}$, i.e., $\mathrm{Ext}_{\text {RoL }}(\mathrm{F}, \mathrm{A})$ is a subfunctor with respect to the second variable.

Now let us prove functorness with respect to the first variable. Let $v: N \rightarrow F$ be an arbitrary homomorphism Then taking $C^{\prime}=v^{-1}(C)$ and $B^{\prime \prime}=\mu^{-1}(B)$ we have the following commutative diagram with exact rows and columns:

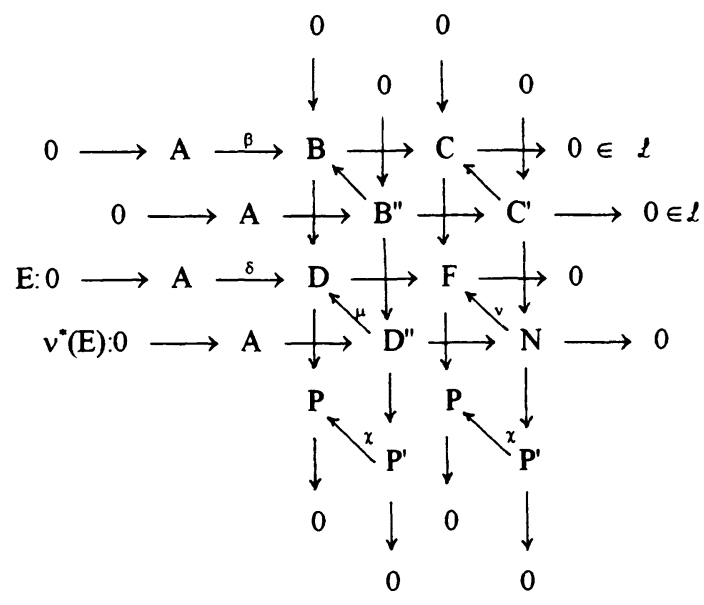


$\chi$ is a monomorphism by Lemma 3.1 . Since $P$ is closed under submodules, $P^{\prime} \cong \operatorname{Im} \chi \in \sigma$ Therefore, by $(\mathrm{P}-1)$ we have $0 \longrightarrow \mathrm{B}^{\prime \prime} \longrightarrow \mathrm{D}^{\prime \prime} \longrightarrow \mathrm{P}^{\prime} \longrightarrow 0 \in \bar{k}(\mathcal{P})=\mathbb{R}$. Since $\mathrm{Ext}_{\perp}(\mathrm{C}, \mathrm{A})$ is a contravanant functor with respect to the first variable, $0 \longrightarrow A \longrightarrow B^{\prime \prime} \longrightarrow C^{\prime} \longrightarrow 0 \in \mathcal{L}$. Thus

$$
v^{*}(\mathrm{E}): 0 \longrightarrow \mathrm{A} \longrightarrow \mathrm{D} \text { " } \longrightarrow \mathrm{N} \longrightarrow 0 \in \mathbb{R} \circ \mathcal{L} .
$$

So $\mathrm{Ext}_{\mathrm{RoL}_{1}}$ is a subfunctor of Ext:R-mod $\times \mathrm{R}-\bmod \rightarrow$ Set.

To prove that $\operatorname{Ext}_{\text {aot }}(C, A)$ is a subgroup of $\operatorname{Ext}(C, A)$ for every $C, A$, let $E_{1}, E_{2} \in E_{x t_{\text {Rot }}}(C, A)$ Since $E_{1}+E_{2}=\nabla_{A}\left(E_{1} \oplus E_{2}\right) \Delta_{C}$ (see Mac Lane [2]), to prove that $E_{1}+E_{2} \in \mathbb{R} \circ$ L, it is sufficient to show that $\mathrm{E}_{1} \oplus \mathrm{E}_{2} \in \mathbb{R} \circ$ L L $\quad$ Let $\quad \mathrm{E}_{1}: 0 \longrightarrow \mathrm{A} \stackrel{\alpha}{\longrightarrow} \mathrm{B}_{1} \longrightarrow \mathrm{C} \longrightarrow 0$ and $\mathrm{E}_{2}: 0 \longrightarrow \mathrm{A} \stackrel{\beta}{\longrightarrow} \mathrm{B}_{2} \longrightarrow \mathrm{C} \longrightarrow 0$ Then $\alpha=\alpha_{2} \circ \alpha_{1}, \quad \beta=\beta_{2} \circ \beta_{1}$, where $\alpha_{1}, \beta_{1}$ are -monomorphisms and $\alpha_{2}, \beta_{2}$ are $\mathbb{Q}$-monomorphisms Clearly, $\alpha_{1} \oplus \beta_{1}$ is an 2 -monomorphism and $\alpha_{2} \oplus \beta_{2}$ is an $a$-monomorphism. Therefore, $\alpha \oplus \beta=\left(\alpha_{2} \oplus \beta_{2}\right) \circ\left(\alpha, \oplus \beta_{1}\right)$ is an $\mathbb{R}_{0} \mathcal{L}$-monomorphism. So $\mathrm{E}_{1} \oplus \mathrm{E}_{2} \in \mathbb{R} \circ \mathcal{L}$.

One can easily show that $E \in \mathbb{R} / \mathcal{L}$ implies $-E \in \mathbb{R} O \mathcal{L}$. Thus $E \times t_{\text {Rol }}$ is a subfunctor of Ext : R-mod x R-mod $\rightarrow \mathrm{Ab}$.

REMARK. Now to prove that $\mathbb{R}_{0} \mathcal{L}$ is a proper class it remains only to show that the composite of two Rol-monomorphisms is an RoL-monomorphism (see Nunke [9]). By means of this fact we have obtained the description of the smallest proper class with given classes of coprojective and coinjective modules as a "composite" of coinjectively and coprojectively generated classes, which will soon be published.

Since $\mathbb{R} \subseteq \mathbb{R} \mathcal{L}$ and $\mathcal{L} \subseteq \mathbb{R} \mathcal{L}$, Lemma 3.2 gives the following inclusion.

THEOREM 3.1. If the class $P$ of modules is closed under extensions and submodules (see [7], [8]), then $\mathbb{R} * \mathcal{L} \subseteq \mathbb{R} \circ \mathcal{L}$ for $\mathbb{R}=\bar{k}(\mathbb{P})$ and for every proper class $\mathcal{L}$.

\section{SOME EXAMPLES IN THE CATEGORY OF ABELIAN GROUPS.}

Let $s_{0}$ be the class of all splitting short exact sequences, \& be the class of all pure short exact sequences and $D$ be the class of all torsion splitting short exact sequences (see Fuchs [1]). For a proper class $\mathbb{Q}$ put

$$
\hat{a}=\{E: \text { for some } n \in Z(n \neq 0) n E \in \mathbb{Q}\} \text {, }
$$

(see Alizade [6]).

Class $\hat{\iota}_{0}$ of all short quasi-splitting exact sequences was introduced and studied by Walker C.P.[10] Classes $\hat{S}$ and $\hat{A}$ were studied by Hart in [11], where these classes were denoted by $\hat{A}$ and $\hat{C}$.

Clearly, $Q+\hat{b_{0}} \subseteq \hat{\mathscr{Q}}$ for every proper class $\mathbb{Q}$.

THEOREM 4.1. $a+\hat{s}_{0}=\hat{a}$.

PROOF. Denote $\mathfrak{Q}+\hat{\vartheta}_{0}$ by g. Every $E \in \mathbb{R}$ can be written as $E=1 \cdot E(n=1)$, therefore $E \in \hat{\mathbb{Q}}$ and then $\mathbb{a} \subseteq \hat{\mathscr{Q}}$ On the other hand, $s_{0} \subseteq \mathbb{Q}$, therefore $\hat{s_{0}} \subseteq \hat{\mathbb{Q}}$. Thus $\mathbb{Q}+\hat{s}_{0} \subseteq \hat{\mathbb{Q}}$.

Conversely, let $\mathrm{E}: 0 \longrightarrow \mathrm{A} \longrightarrow \mathrm{B} \longrightarrow \mathrm{C} \longrightarrow 0 \in \hat{\mathfrak{R}}$. Then $\mathrm{nE} \in \mathbb{R}$ for some $n \neq 0$. Let us denote by $n$ the endomorphism of multiplication by $n$ on $A$. Then by Lemma 52.1 in Fuchs [1] $n E$ is the lower exact sequence in the following commutative diagram:

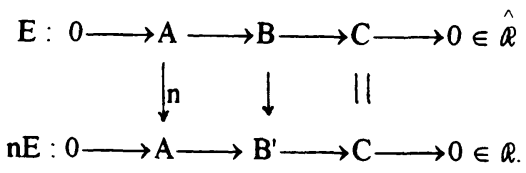


The endomorphism $n: A \rightarrow A$ can be represented in the natural way $n=\alpha \circ \sigma$, where $\sigma . A \rightarrow n A$ is epimorphism and $\alpha: \mathrm{nA} \rightarrow \mathrm{A}$ is inclusion map

We have the following commutative diagrams with exact rows and columns:

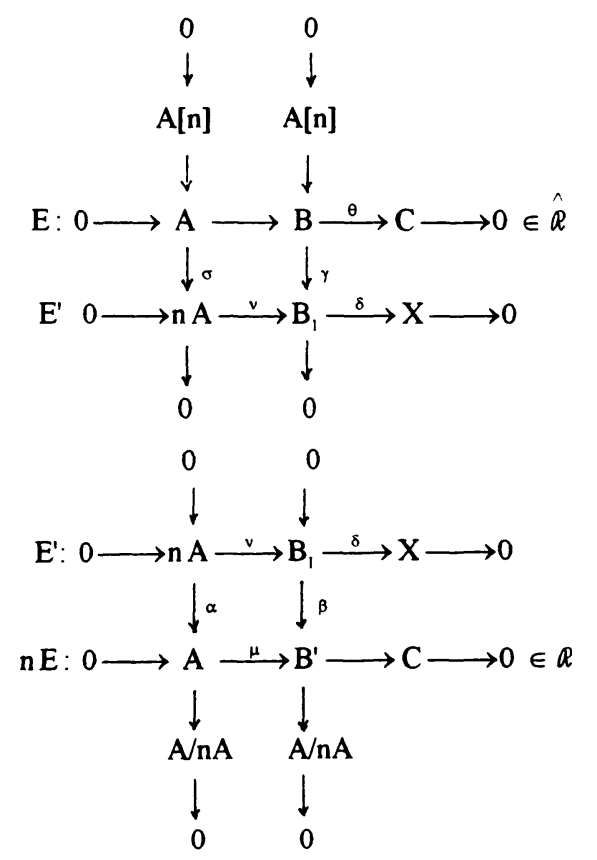

$\mathrm{A} / \mathrm{nA}$ is a bounded and hence $\hat{s_{0}}$-coprojective group. Therefore the short exact sequence $0 \longrightarrow \mathrm{nA} \stackrel{\alpha}{\longrightarrow} \mathrm{A} \longrightarrow \mathrm{A} / \mathrm{nA} \longrightarrow 0$ belongs to $\hat{s}_{0}$ and since $\hat{s}_{0} \subseteq 9$, it belongs to J, 1 e., $\alpha$ is a $J$-mono-morphism. Since $n E \in \mathbb{R} \subseteq \mathcal{I}, \mu$ is a $\mathcal{g}$-monomorphism. Therefore, by $(P-3), \mu \circ \alpha=\beta \circ v$ is a $\mathcal{J}$ monomor-phism. Hence, by $(\mathrm{P}-4), v$ is a 9 -monomorphism. Thus $\mathrm{E}^{\prime} \in \mathcal{J}$.

$\mathrm{A}[\mathrm{n}]$ is a bounded and therefore $\hat{b}_{0}$-coinjective group. Hence the short exact sequence $0 \longrightarrow \mathrm{A}[\mathrm{n}] \longrightarrow \mathrm{B} \longrightarrow \mathrm{B}_{1} \longrightarrow 0$ belongs to $\hat{s}_{0}$ and since $\hat{s_{0}} \subseteq 9$, it bélongs to 9 , i e. $\gamma$ is a I-epimorphısm. Since $E^{\prime} \in \mathcal{I}, \delta$ is a $\mathcal{I}$-epimorphism. By $\left(\mathbf{P}^{\prime} 3^{\prime}\right) \theta=\delta \circ \gamma$ is a $\mathcal{I}$-epimorphism. Therefore $E \in \mathcal{J}$ and we have $\hat{a} \subseteq \mathcal{J}$.

DEFINITION. For a subgroup $A$ of $B$ let $\hat{A}=\{b \in B: m b \in A$ for some $m \in Z,(m \neq 0)\}$

LEMMA 4.1. Let $B_{d}$ be the maximal divisible subgroup and $T(B)$ be the torsion part of a group $B$. Then

$$
\hat{B_{d}}=B_{d}+T(B)=B_{d}+\hat{0}
$$

PROOF. The inclusion $B_{d}+T(B) \subseteq \hat{B_{d}}$ is obvious.

Let $B=B_{d} \oplus K, K \leq B$ and let $b=a+k$ be any element from $\hat{B}_{d}\left(a \in B_{d}, k \in K\right)$. Then $n b \in B_{d}$ for some $n \neq 0$. Since $B_{d}$ is divisible, $n b=n a_{1}$ for some $a_{1} \in B_{d}$. Therefore $n a+n k=n b=n a_{1}$. By directness of the sum $B_{d} \oplus K, n k=0$ and hence $k \in T(B)$. So $b=a+k \in B_{d}+T(B)$

Now we take $B=\operatorname{Ext}(C, A)$ for any groups $C, A$, then $B_{d}=\operatorname{Ext}_{g}(C, A)$ by Prop. 58.3 in Fuchs $[1]$ and $T(B)=\operatorname{Ext}_{\hat{\phi_{0}}}(C, A)$ (see $\left.[10]\right)$. Therefore by Lemma 4.1 we have 


$$
\operatorname{Ext}_{\hat{\hat{b}}}(\mathrm{C}, \mathrm{A})=\mathrm{Ext}_{\boldsymbol{\partial}}(\mathrm{C}, \mathrm{A})+\mathrm{Ext}_{\hat{\hat{\phi}_{0}}}(\mathrm{C}, \mathrm{A})
$$

for each group C,A, i.e., $\hat{A}=\$ * \hat{b}_{0}$. This equality together with Theorem 3.1 (note that $D=\overline{\mathbf{k}}(P)$, $\rho$ being the class of all torsion free groups (see [7]) gives us the following theorem.

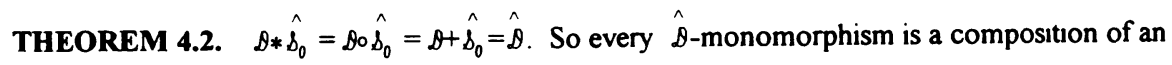
$\hat{\hat{h}_{0}}$-monomorphism and a $\mathscr{A}$-monomorphism.

For the class $\&$ the situation is worse.

LEMMA 4.2. There exists a reduced cotorsion group $C$, Ulm's subgroup of which is torsion free and algebraically compact group

PROOF Let $J_{p}$ be the group of $p$-adic numbers. The $p$-basic subgroup $B=<b>$ of $J_{p}$ is isomorphic to the group $Z$ of integers and $J_{p} / B$ is a divisible group (see ch.20 and 32 in Fuchs [1]). Let us construct an extension $K$ of $B$ such that the first Ulm's subgroup $K^{\prime}$ of $K$ is equal to $B$. Define $K$ by means of generators

$$
b_{1}, b_{2}, \ldots, b_{n}, \ldots: K=<b_{1}, b_{2}, \ldots, b_{n}, \ldots>
$$

and relations

$$
b_{1}=b, 2 b_{2}=b, \quad, n b_{n}=b, \ldots
$$

$K / B$ is isomorphic to a direct sum of cyclic groups, hence $(K / B)^{1}=0$ and we have $K^{\prime} \subseteq B$. On the other hand, the inclusion $B \subseteq K^{\prime}$ is obvious and thus $K^{\prime}=B$.

Let us complete the diagram

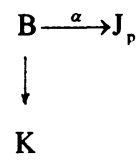

to a pushout diagram. Then we have the following commutative diagram with exact rows and columns:

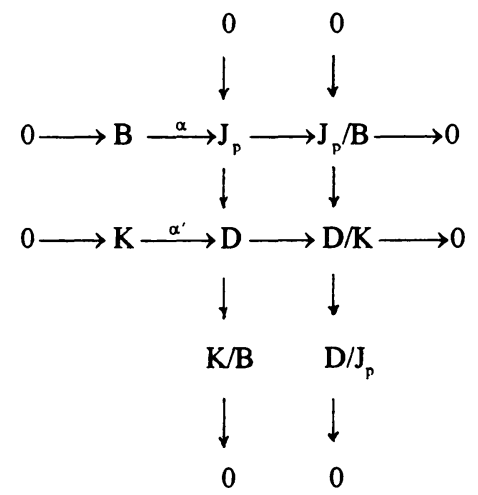

Since $\alpha$ is a monomorphism, $\alpha$ is also a monomorphism and $J_{p} / B \cong D / K, K / B \cong D / J_{p}$ (see Th $10.2[1]$ ). Now let us prove that $D^{\prime}=J_{p}$. Since $\left(D / J_{p}\right)^{\prime} \cong(K / B)^{\prime}=0$ we have $D^{\prime} \subseteq J_{p}$. On the other hand let $x \in J_{p}$ and $n \in Z(n \neq 0)$ be arbitrary. Since $J_{p} / B$ is divisible, there is $x_{1} \in J_{p}$ such that $x-n x_{1} \in B \quad B=K^{1}$, 
therefore there is an element $k \in K$ such that $x-n x_{1}=n k$. Then $x=n\left(x_{1}+k\right) \in n D$ Thus we have $x \in D^{\prime}$ and $\mathrm{D}^{\prime}=\mathrm{J}_{\mathrm{p}}$

Since $\mathrm{D} / \mathrm{J}_{\mathrm{r}}$ is isomorphic to a direct sum of finite cyclic groups, it can be embedded into a direct product of these cyclic groups, i.e., into a reduced algebraically compact group $M \cdot D / J_{\Gamma} \stackrel{\beta}{\longrightarrow} M$. By Theorem $51.3[1]$

$$
\beta^{*}: \operatorname{Ext}\left(M, J_{p}\right) \longrightarrow \operatorname{Ext}\left(D / J_{p}, J_{p}\right)
$$

is an epımorphism. Therefore there is an extension $C$ of $D$ such that the following diagram with exact rows and columns is commututive:

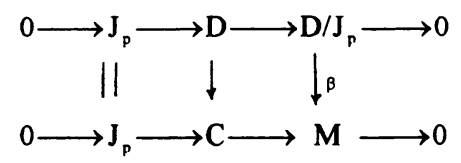

$J_{\mathrm{p}}=D^{\prime} \subseteq C^{\prime}$. Since $M$ is a reduced algebraically compact group, we have $\left(C / J_{p}\right)^{\prime} \cong M^{\prime}=0$ by Prop.54.2 [1]. Then $C^{\prime} \subseteq J_{p}$. Hence we have $C^{\prime}=J_{p}$ and $C$ is a reduced group. By Prop. 394 [1] $J_{p}$ is an algebraically compact group. Since $\mathrm{J}_{\mathrm{p}}$ and $\mathrm{M}$ are algebraically compact and hence cotorsion groups, by ch 54(D) [1] $\mathrm{C}$ is a reduced cotorsion group.

LEMMA 4.3. If the Ulm's subgroup $A=C^{\prime}$ of a reduced group $C$ is torsion free and different from zero, then

$$
\mathrm{A}+\mathrm{T}(\mathrm{C}) \neq \hat{\mathrm{A}}
$$

$\mathrm{T}(\mathrm{C})$ being the torsion part of $\mathrm{C}$.

PROOF. Take an element $a \notin n A$ of the reduced group $A$. Since $A=\underset{0 \neq n \in Z}{\cap} n C$, there is $b \in C$ such that $n b=a$. Obviously $b \in \hat{A}$. If $b \in A+T(C)$, one can write $b=a_{1}+t$ with $a_{1} \in A$ and $t \in T(C)$. Then $a=n b=n a_{1}+n t$ and hence $n t=a-n a_{1} \in A \cap T(C)$. Since $A$ is torsion free, $n t=0$ and we have $a=n a_{1} \in n A$ But this contradicts the assumption. Thus $b \in \hat{A} \backslash(A+T(C))$.

The following theorem demonstrates that for proper classes $\mathbb{Q}$ and $\mathcal{L}$ the class $\mathbb{Q} * \mathcal{L}$ need not be proper.

THEOREM 4.3. $\diamond * \hat{\delta}_{0} \neq \hat{\delta}$.

PROOF. According to Lemma 4.2 there is a reduced cotorsion group $C$, Ulm's subgroup $A=C^{\prime}$ of which is reduced, torsion free and algebraically compact. By ch. $54(\mathrm{H})[1]$ we have

$$
\operatorname{Ext}(\mathbf{Q} / \mathbf{Z}, \mathbf{C}) \cong C
$$

By Theorem $53.3[1]$

$$
\operatorname{Ext}_{b}(\mathbf{Q} / \mathbf{Z}, \mathrm{C})=\operatorname{Pext}(\mathbf{Q} / \mathbf{Z}, \mathrm{C}) \cong \mathrm{C}^{\prime}=\mathrm{A}
$$

On the other hand,

$$
\operatorname{Ext}_{\hat{\phi}_{0}}(\mathbf{Q} / \mathbf{Z}, C)=\operatorname{Text}(\mathbf{Q} / \mathbf{Z}, C) \cong T(C)
$$

and

$$
\operatorname{Ext}_{\hat{\imath}}(\mathbf{Q} / \mathbf{Z}, \mathbf{C}) \cong \hat{\mathbf{A}}
$$


By Lemma 43 we have

$$
E \times t_{s}(Q / Z, C)+E x t_{\hat{\phi_{0}}}(Q / Z, C) \neq \operatorname{Ext}_{\hat{s}}(Q / Z, C)
$$

Thus $s * \hat{\iota}_{0} \neq \hat{\&}$.

COROLLARY 4.1. The class $\diamond * \hat{\iota}_{0}$ is not proper

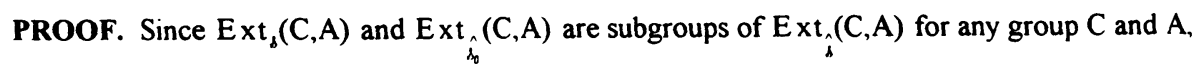
evidently $s * \hat{\iota}_{0} \subseteq \hat{\delta}$. Since $\hat{s}=\delta+\hat{\wp}_{0}$ is the least proper class including $s$ and $\hat{\delta}_{0}$, if $s * \hat{b}_{0}$ were a proper class we should have $\hat{\delta} \subseteq \delta * \hat{\delta}_{0}$ and hence $\delta * \hat{\delta}_{0}=\hat{\delta}$. But this contradicts Theorem 4.3.

ACKNOWLEDGEMENT. The author wishes to express his gratitude to his referee for helpful critıcism.

\section{REFERENCES}

[1] FUCHS, L., "Infinite abelian groups," v.1, Academic Press, 1970

[2] MAC LANE, S., "Homology," Springer-Verlag, 1975.

[3] BUCHSBAUM, D., "A note on homology in categories" Ann. of Math. 69:1(1959), 66-74.

[4] SKLYARENKO, E.G., "Relative homological algebra in the category of modules," Usp. Mat. Nauk (Russian Math. Surveys), 33:3(1978), 85-120.

[5] STENSTRÖM, B., "Pure submodules," Arkiv Math. 7 12(1967), 159-171.

[6] ALIZADE, R.H., "On the proper classes of short exact sequences in the category of abelian groups," Math. Notes 40:1(1986), 3-15.

[7] ALIZADE, R.H., "On the proper classes of Kepka," Math. Notes 37:2(1985), 268-273

[8] KEPKA, T, "On one class of purities," Comment. Math. Univ. Carol. 14:1(1973), 139-154.

[9] NUNKE, R.J., "Purity and subfunctors of the identity," - In: Topics in abelian groups; (icago, $1963,121-171$.

[10] WALKER, C.P. "Properties of Ext and quasi-splitting of abelian groups," Acta Math. Acad. Scl. Hung., 15(1964), 157-160.

[11] HART, N., "Two parallel homological algebras," Acta Math. Acad. Sci. Hung., 25:3-4(1974), 321-327 


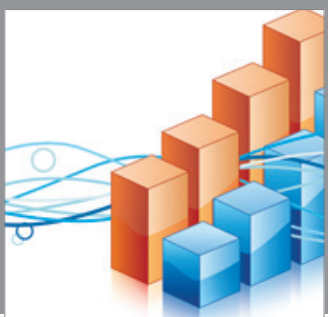

Advances in

Operations Research

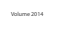

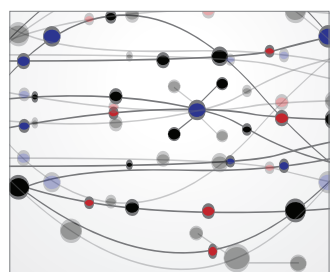

\section{The Scientific} World Journal
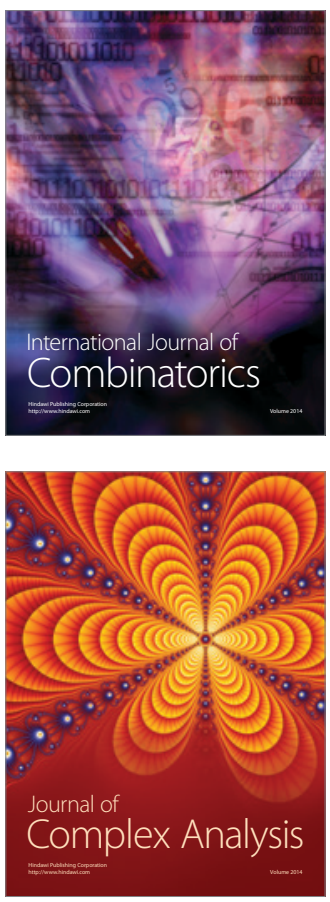

International Journal of

Mathematics and

Mathematical

Sciences
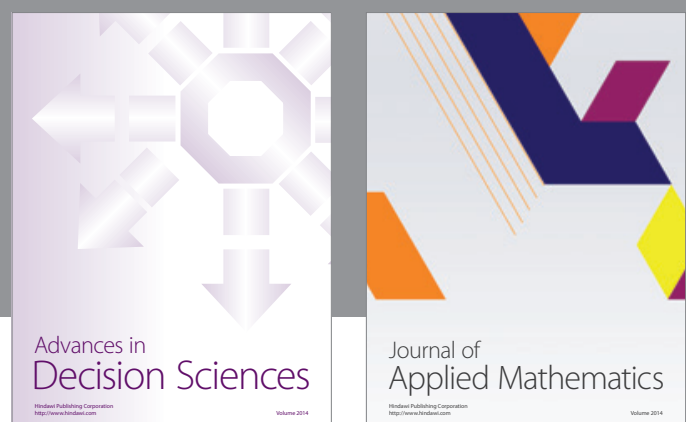

Journal of

Applied Mathematics
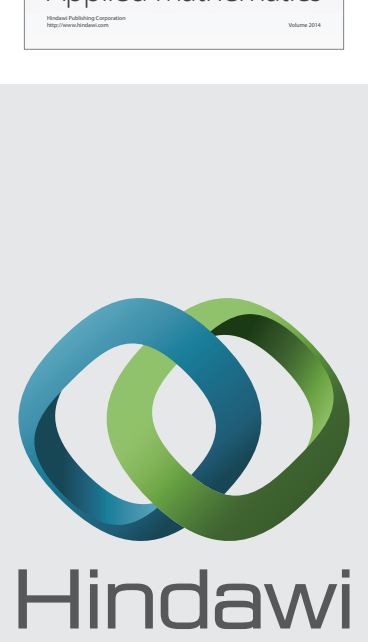

Submit your manuscripts at http://www.hindawi.com
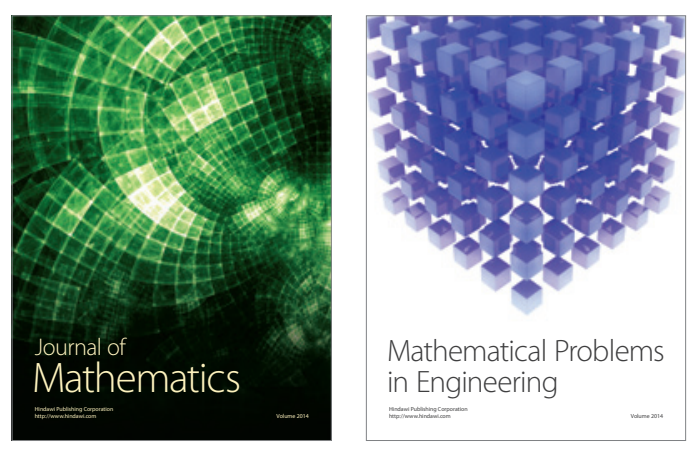

Mathematical Problems in Engineering
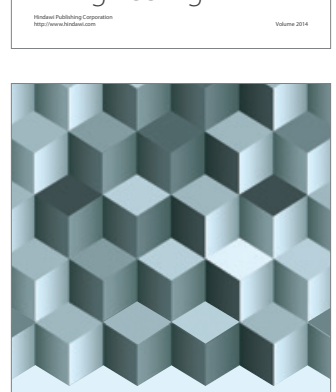

Journal of

Function Spaces
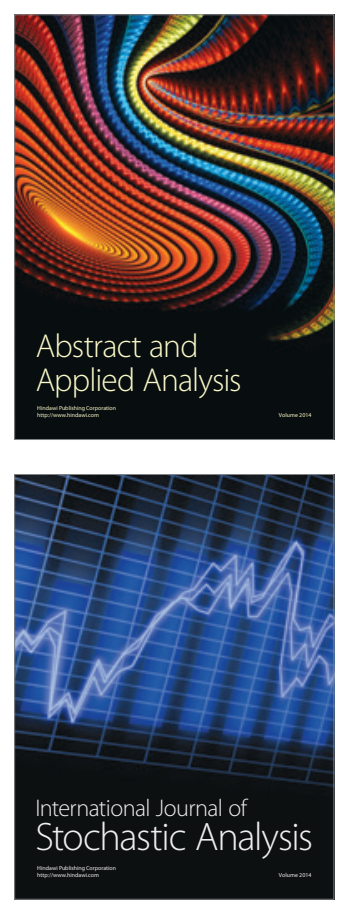

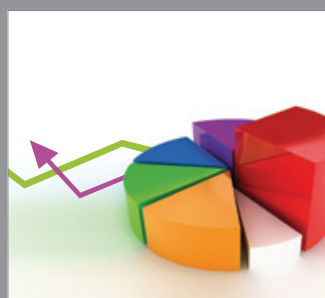

ournal of

Probability and Statistics

Promensencen
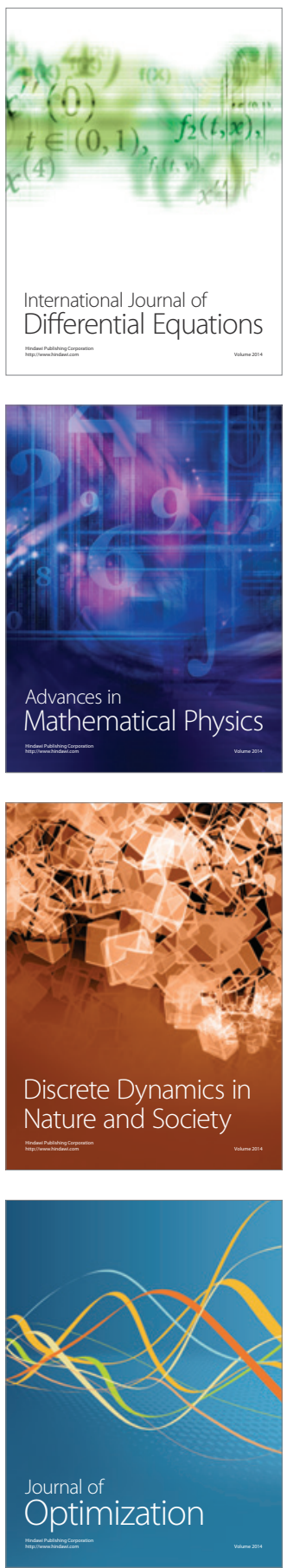